\title{
Estruturação de um Sistema Computacional (SAD) para avaliação da Gestão do Conhecimento
}

\author{
Wagner Igarashi (UEM) wigarash@gmail.com \\ Debora Donadel dos Santos (UNIOESTE) deboradonately@gmail.com \\ Deisy Cristina Corrêa Igarashi (UEM) deisyigarashi@gmail.com
}

\begin{abstract}
Resumo. A avaliação de desempenho é uma atividade essencial vinculada ao processo de Gestão do Conhecimento (GC). O estudo estruturou um sistema computacional (SAD) para apoiar a avaliação de GC. O SAD permite estruturar modelos de avaliação de GC, inserir o desempenho de cada indicador, bem como mostrar graficamente os resultados parciais e finais de uma avaliação. A experimentação do SAD foi realizada em uma organização de ensino à distância.
\end{abstract}

Palavras chave: sistema computacional, avaliação, gestão do conhecimento.

\section{Structuring of the Computer System (SAD) for Knowledge Management evaluation}

\begin{abstract}
The performance evaluation is an essential activity related to Knowledge Management (KM) process. The study has structured a computer system (SAD) to support KM evaluation. The SAD implementation allows structure KM evaluation models, insert the performance of each indicator, as well as graphically show partial and final results of an evaluation. The SAD experimentation was accomplished in a distance learning organization.
\end{abstract}

Keywords: computer system, evaluation, knowledge management.

\section{Introdução}

Devido a concorrência nos mercados, as organizações têm buscado cada vez mais informações que as auxiliem a direcionar esforços de aprendizagem e de gestão do conhecimento, a fim de melhorar seus desempenhos. Para tanto os gestores necessitam de informações que os capacitem a exercer julgamentos baseados em fatos. Tais informações constituem elementos que apoiam a Gestão do Conhecimento (GC) nas organizações (Drucker, 2000; Santos et al., 2007).

Deming (2003) destaca que não é possível gerenciar o conhecimento sem primeiramente medi-lo. Contudo, não se consegue medir o conhecimento sem antes se ter um processo de avaliação. Por isso, os gestores necessitam de informações que os capacitem a exercer julgamentos baseados em fatos. Isso exige quatro segmentos de informações de diagnóstico: básicas, produtividade, competências e sobre alocação de recursos. Estas informações constituem os elementos fundamentais que apoiam a Gestão do Conhecimento (GC) nas organizações (Drucker, 2000).

Além da avaliação em si, é indispensável que a organização obtenha um suporte tecnológico para executar de modo contínuo e sistemático a GC. Isto se justifica pela quantidade de informação relevante, pela necessidade de agilidade no processo, segurança e praticidade. Tais fatores justificam a utilização de um sistema de informação computacional para viabilizar a avaliação da GC nas organizações.

Esta por sua vez, devido a quantidade de informação envolvida e a necessidade 
de agilidade, segurança e praticidade pode ser apoiada pelo desenvolvimento de sistemas computacionais que viabilize a avaliação da GC nas organizações.

No contexto relatado, o presente estudo tem por objetivo estruturar um sistema informação computacional para apoiar a avaliação da GC, em um modelo que contempla aspectos como: estratégia organizacional, cultura organizacional, estrutura organizacional, recursos humanos internos e relações externas.

\section{Trabalhos relacionados}

$\mathrm{Na}$ literatura foram encontradas diversas definições sobre gestão do conhecimento, dentre as quais se destacam as definições elencadas no Quadro 1.

Quadro 1 - Definições sobre o termo GC

\begin{tabular}{|c|c|}
\hline (Davenport e Prusak, 1998) & $\begin{array}{l}\text { A gestão do conhecimento deve contribuir na geração, codificação, } \\
\text { transferência e utilização do conhecimento existente na organização }\end{array}$ \\
\hline (Sveiby, 1998) & $\begin{array}{l}\text { A gestão do conhecimento é uma ferramenta capaz de agregar valor aos } \\
\text { ativos da organização }\end{array}$ \\
\hline (Tarapanoff, 2001, p. 137) & $\begin{array}{l}\text { "a gestão do conhecimento pode ser vista como o conjunto de atividades } \\
\text { que busca desenvolver e controlar todo tipo de conhecimento em uma } \\
\text { organização, visando á utilização na consecução de seus objetivos" }\end{array}$ \\
\hline (Terra e Gordon, 2002, p. 57) & $\begin{array}{l}\text { "a gestão do conhecimento, em seu sentido mais atual, pode ser } \\
\text { considerada o esforço para melhorar o desempenho humano e } \\
\text { organizacional por meio de facilitações de conexões significativas" }\end{array}$ \\
\hline (Igarashi et al., 2008, p. 2) & $\begin{array}{l}\text { "a gestão do conhecimento organizacional refere-se a um processo de } \\
\text { mudança cultural, sendo a aprendizagem organizacional um elemento } \\
\text { essencial, que deveria ser apoiado por ferramentas de tecnologia da } \\
\text { informação" }\end{array}$ \\
\hline
\end{tabular}

Deming (2003) destaca que em uma organização não é possível gerenciar o conhecimento sem primeiramente medi-lo, não se consegue medir o conhecimento sem antes se ter um processo de avaliação de desempenho. Por isso, os gestores necessitam de informações que os capacitem a tomar decisões baseados em fatos.

Em síntese, Igarashi et al. (2010) consideram que o processo de avaliação em si não gera os benefícios efetivamente esperados, a menos que a ele esteja atrelado aos elementos vinculados à medição e ao gerenciamento do conhecimento. Neste sentido, a avaliação pode ser percebida como uma atividade que auxilia a direcionar os esforços de aprendizagem e GC, principalmente se apoiada por sistemas computacionais.

Atualmente os sistemas computacionais encontram-se diante do desafio de proporcionar aos tomadores de decisão a apresentação de informações confiáveis, precisas, oportunas e relevantes (Tait, 2000). Nesse contexto, tais sistemas podem tornar-se úteis ao processo decisório, possibilitando coletar, processar e armazenar dados, de modo a obter informações que satisfaçam aos requisitos mencionados.

Com este enfoque, ferramentas computacionais para GC podem ser classificadas em seis grupos principais: ferramentas voltadas para internet, sistemas de gerenciamento eletrônico de documentos, sistemas de groupware, sistemas de workflow, sistemas de mapas de conhecimento e sistemas para descoberta de conhecimento em bases de dados (Carvalho, 2000; Liao, 2003; Rouse e Sage, 2007, Rouse e Compton, 2009; Berzisa e Grabis, 2011). Esta classificação não é exaustiva e nem conclusiva. Outro enfoque adota sistemas computacionais direcionados à avaliação de GC (Igarashi et al., 2012), foco do presente estudo.

Para a identificação da existência de pesquisas similares, no contexto nacional, 
realizou-se uma revisão sistemática junto à base de dados SCiELO - Scientific Eletronic Library Online. A pesquisa resultou em 27 artigos, dos quais foram selecionados 9, pois 12 artigos eram repetidos e 6 artigos estavam fora do escopo.

Portanto, nos 9 artigos foi realizada uma leitura detalhada que possibilitou a coleta de dados e características de cada tema individualmente. $\mathrm{Na}$ análise individual verificou-se que nenhum dos artigos tratava de uma proposta de avaliação de desempenho organizacional, no sentido de qualquer organização poder utilizar um modelo ou software (Salomi, Miguel e Abackerli, 2005; Guimarães et al., 2007; Lins et al., 2007; Mello et al., 2009). Os artigos tratam de avaliações mais específicas, como por exemplo, avaliação de colaboradores, avaliação de hospital, levantamento de indicadores para um caso específico ou avaliação de processos e equipamentos.

\section{Metodologia}

\subsection{Requisitos do sistema}

Dentre as funcionalidades levantadas mediante a análise de requisitos e que forneceram suporte para a especificação do sistema destacam-se: (a) a modelagem de indicadores de desempenho segundo os objetivos estratégicos, tornando o sistema adaptável a diferentes organizações; (b) mecanismo de carga dos pesos para cada nível do modelo de GC; (c) visualização gráfica da avaliação de desempenho; (d) controle de acesso; (e) armazenamento de dados em meio não volátil e seguro.

\subsection{Ferramentas utilizadas}

Para o desenvolvimento do sistema foram utilizadas ferramentas de software livre. Na fase de projeto do sistema optou-se por utilizar a modelagem de sistemas orientada a objetos (Sommerville, 2007), com linguagem UML (Unified Modeling Language) (Booch et al., 2006). O suporte ao processo de modelagem em UML, foi realizada via ferramenta JUDE Community (versão 5.3). Para plataforma de desenvolvimento do sistema utilizou-se a IDE Eclipse (versão 3.4.1), pela quantidade de recursos que a IDE (Integrated Development Environment) apresenta e versatilidade na instalação de plug-ins.

Na codificação do sistema utilizou-se linguagem de programação Java (versão 6), a qual tornou o software portável para diferentes sistemas operacionais. Para a interface gráfica foi utilizada a biblioteca Swing em conjunto com o uso do Jigloo, que permitiu construir e gerenciar as interfaces visualmente. Jigloo é um plug-in que pode ser adicionado ao Eclipse, utilizando as funcionalidades da IDE.

Além do Swing, especificamente para a visualização do modelo, utilizou-se o framework Prefuse, o qual é uma arquitetura para visualização de grafos e árvores (Cormen et al., 2002). A utilização do Prefuse justifica-se pelo fato da estrutura do modelo de GC ser hierárquica e o framework possuir estruturas de dados otimizadas, gerência de layouts de visualização, classes para interação com o grafo e animações.

Para o armazenamento dos dados, foi escolhido o SGBD MySQL (versão 5.1.37). E para criação e documentação do modelo entidade-relacionamento da base de dados (Silberschatz et al., 2006), foi empregado o software DBDesigner 4. 


\section{Projeto do Sistema de Avaliação de Desempenho (SAD)}

O projeto do SAD foi modelado utilizando orientação a objetos. Desta forma, as unidades funcionais e lógicas do software foram definidas através de classes, de modo que as funcionalidades são executadas através da troca de mensagens entre os diversos objetos que compõem o software no momento de sua execução.

Segundo Sommerville (2007), um objeto define-se como sendo: “(...) uma entidade que possui um estado e um conjunto definido de operações definidas para funcionar neste estado. O estado é representado como um conjunto de atributos de objeto. As operações associadas ao objeto fornecem serviços a outros objetos (clientes) que solicitam esses serviços quando alguma computação é necessária".

Todo o objeto é uma instância de uma classe. A classe define quais são os atributos e quais são os métodos que o objeto possui. Desta forma, pode-se entender a classe como uma fôrma que serve de modelo a criação de novos objetos. Ela contém em si toda a especificação da unidade do universo real mapeado. Mais especificamente, a Figura 1 tem o propósito de mapear as classes relativas ao sistema aqui proposto.

Uma das grandes vantagens em se desenvolver um software orientado a objetos é criar estruturas suficientemente genéricas e consistentes com objetos do mundo real. Isso torna o código produzido mais sucinto, compreensível, manutenível e reutilizável.

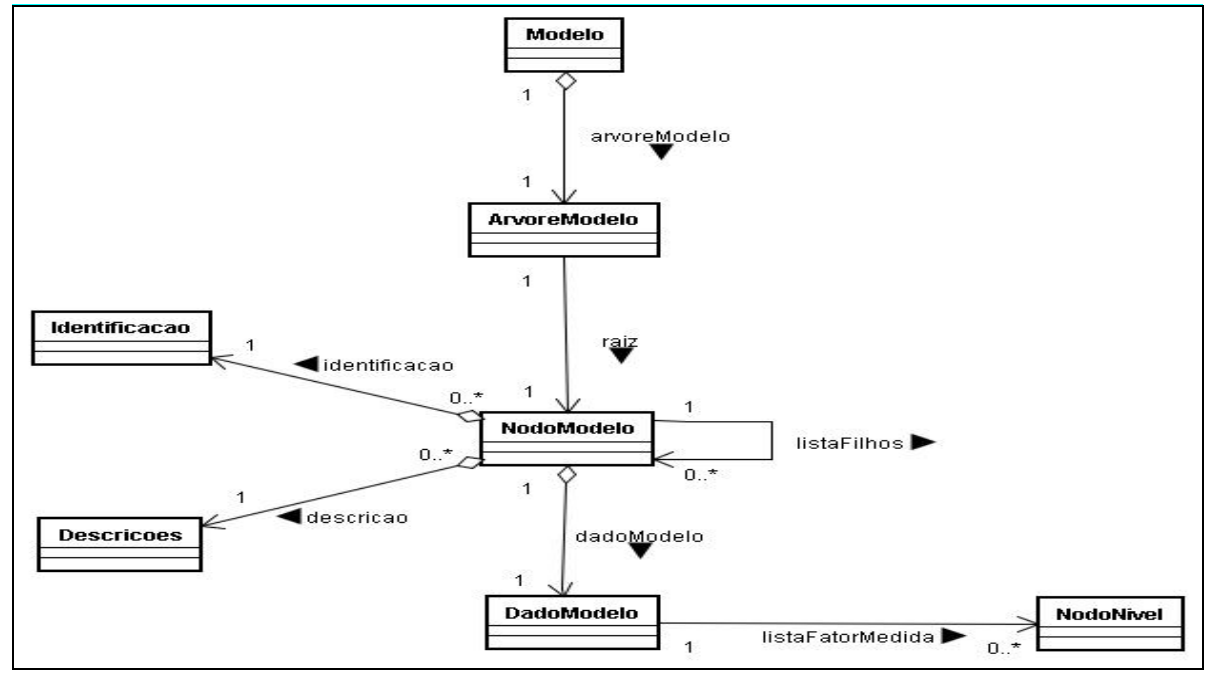

Figura 1 - Diagrama de classes de negócio.

Para fins de armazenamento das informações do SAD foi criado o modelo no formato entidade-relacionamento, Figura 2. O modelo utiliza a sintaxe dos tipos de dados do banco de dados MySQL. Com as especificações, requisitos, modelo de classes e modelo de dados definidos, tem-se base e subsídio para a implementação do sistema. 


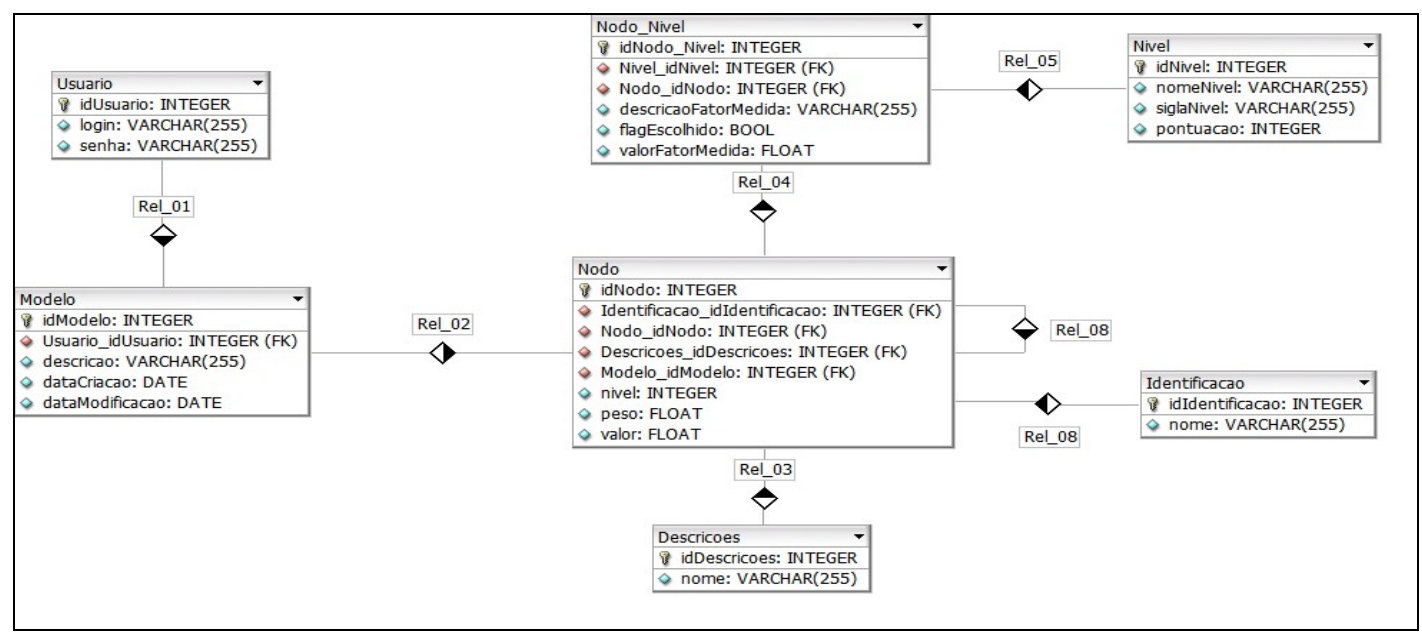

Figura 2 - Modelo de entidade e relacionamento.

\section{Implementação}

$\mathrm{Na}$ implementação do SAD utilizou-se o conceito de camadas de software, o qual auxilia a tarefa de separar responsabilidades, promove um baixo acoplamento e um alto nível de coesão. As camadas de software reduzem a complexidade do sistema, tornando mais simples seu entendimento, elas também promovem a reutilização dos componentes do sistema, devido ao baixo acoplamento proporcionado (Larman, 2007).

Neste aspecto, o SAD possui as seguintes camadas: SADApresentação responsável pela camada de apresentação onde estão as classes das interfaces do sistema; SADNegocio - cuida dos interesses das classes de controle das tabelas do banco de dados e das classes das regras de negócio; SADBO - contém as classes de negócio; SADInfraBD - mantém as classes de conexão com o banco de dados, criação e execução das operações de manipulação das tabelas.

A interface do SAD com o usuário é do tipo desktop, a partir da qual se pode criar um novo modelo ou acessar um previamente criado. Após a criação, o SAD já estabelece 5 percepções, a partir dos quais o usuário poderá adicionar níveis de preocupação, bem como indicadores. Nos níveis de percepção, preocupação e indicadores o usuário deverá atribuir pesos, de modo que o somatório em cada agrupamento hierárquico resulte em 100 pontos, caso isto não seja feito o sistema acusa uma mensagem de erro.

Para ser possível a estruturação, modificação ou até mesmo a remoção do modelo é necessária a recuperação de sua estrutura de dados (árvore) armazenada em banco de dados. Tais ações utilizam recursão (Cormen et al., 2002). Na Figura 3 é possível ver parte da implementação da consulta de um nó do Modelo, por ser uma estrutura hierárquica, na consulta do Modelo será retornada toda a ramificação a partir do nó passado ao método. 


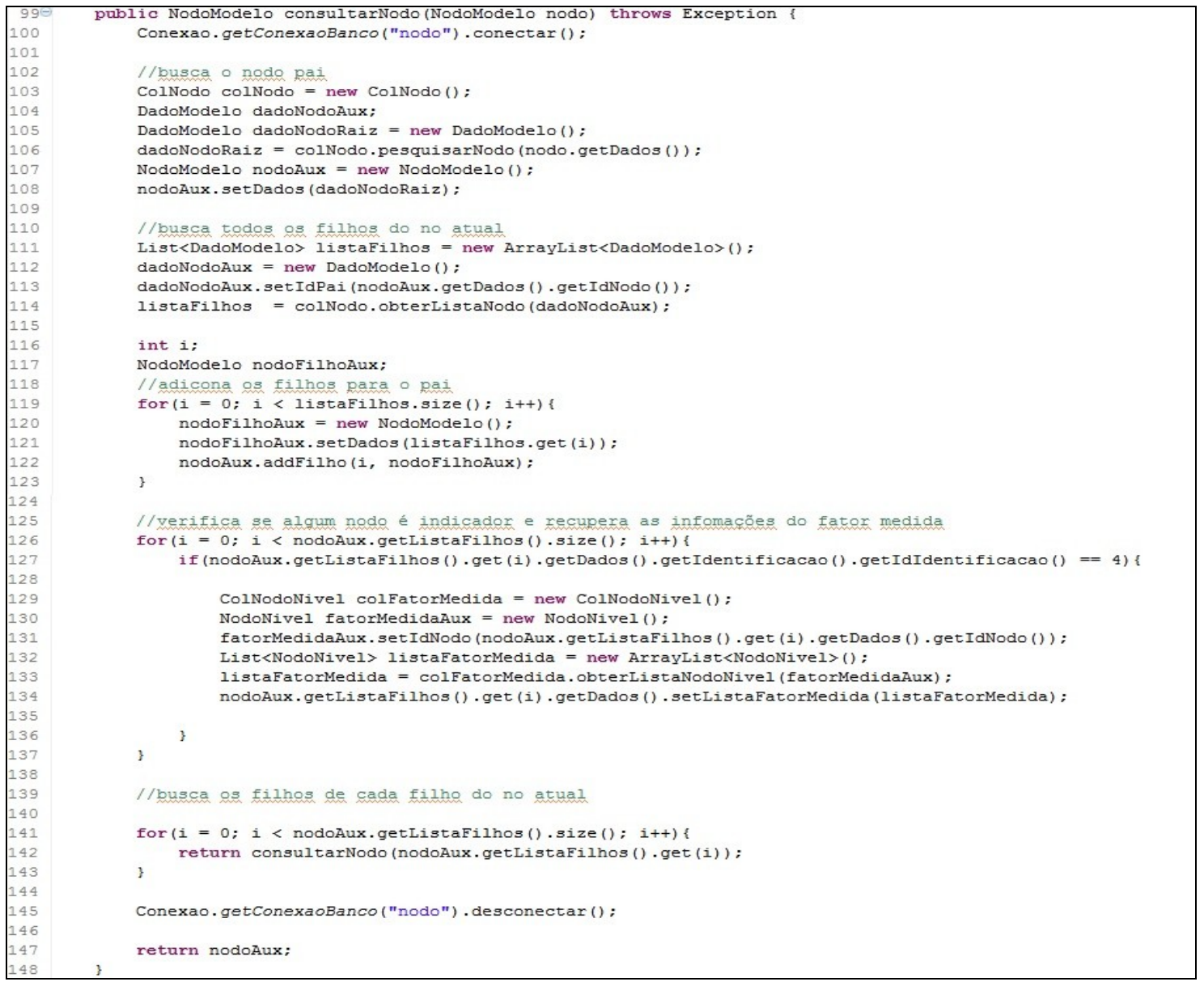

Figura 3 - Código da implementação do método consultar nodo.

Após a estruturação e carga do modelo é possível visualizar o resultado na forma gráfica, utilizando o framework Prefuse que disponibiliza vários tipos de interações, e exige apenas que as informações estejam estruturadas em formato XML, como exemplificado na Figura 4.

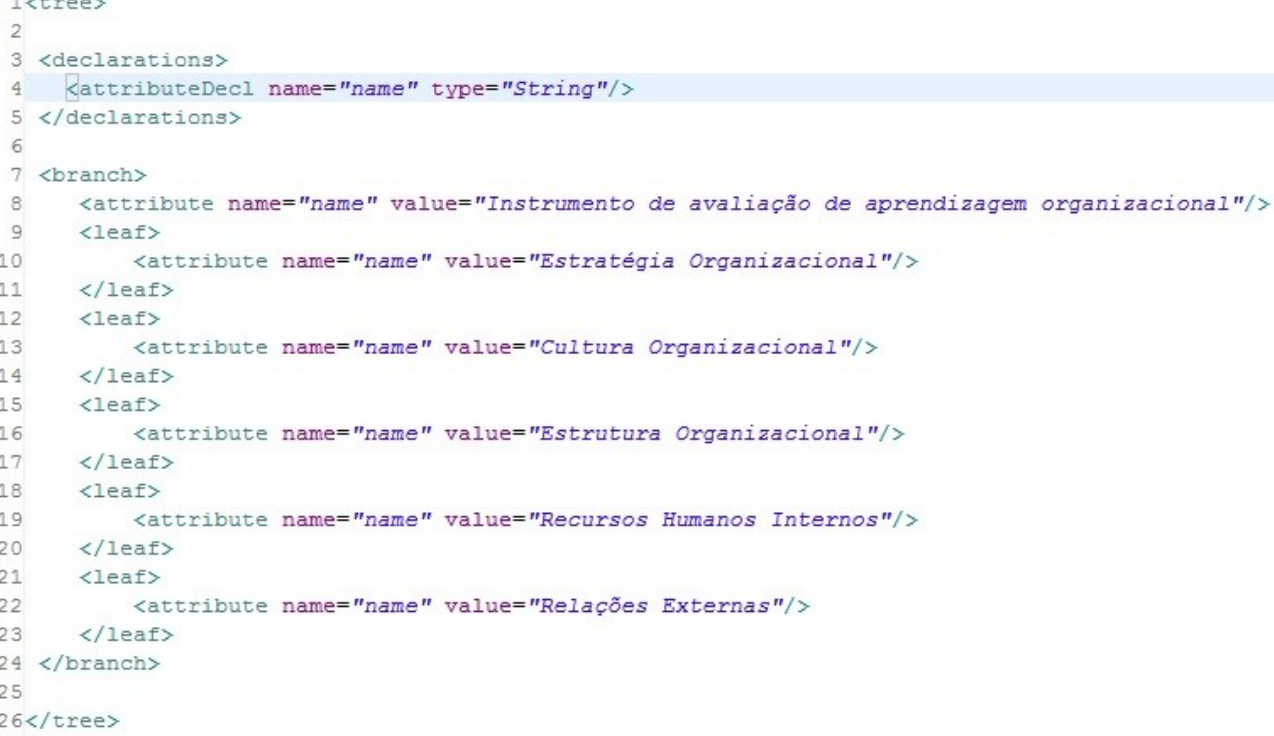

Figura 4 - Estrutura do XML. 


\section{Validação do SAD}

Para validação do sistema computacional utilizou-se as preocupações, indicadores e pesos correlatos referentes à percepção "estrutura organizacional", de uma organização de ensino à distância aqui denominada POLO EAD.

O SAD tem funcionalidade de autenticação, a partir do qual o usuário habilitado abre a interface principal, Figura 5 (a), permitindo criar ou editar um modelo, carregar os indicadores e visualizar o resultado da avaliação. Ao se criar um modelo, é apresentada a tela da Figura 5 (b), onde é possível inserir os pesos de cada percepção, os quais devem ser valores inteiros cuja soma seja igual a 100.

Ressalta-se que o usuário pode estruturar o modelo em etapas, salvando as alterações e editando-o a qualquer momento, Figura 5 (c). Na opção Estruturar/Carregar Indicadores (menu operacionalização do modelo) é possível estruturar o modelo conforme os objetivos organizacionais, na forma de preocupações (níveis intermediários) e indicadores (níveis de base), bem como seus respectivos pesos, Figura 5 (d).

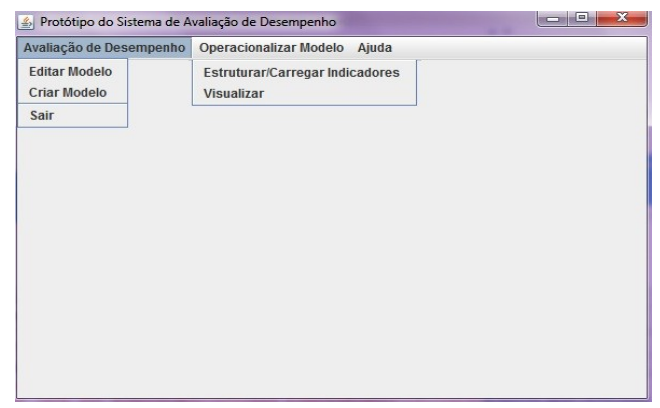

(a) Tela Principal

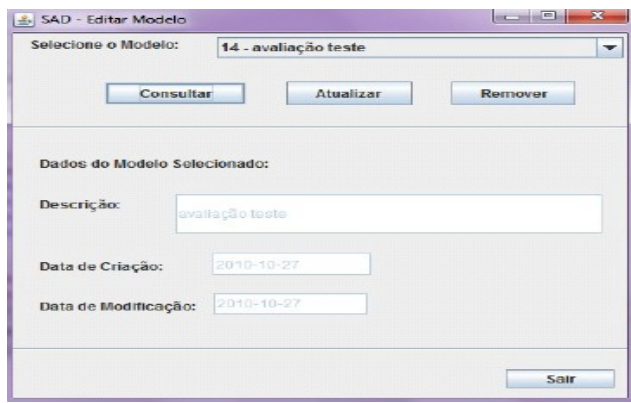

(c) Tela para editar o Modelo.

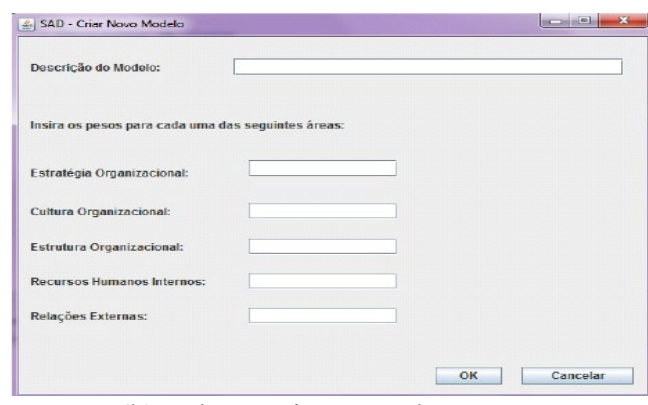

(b) Tela para inserção dos pesos

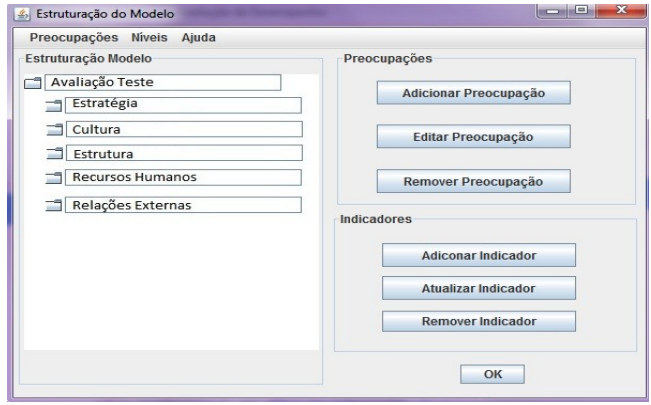

(d) Tela Estruturação do Modelo.

Figura 5 - Telas do sistema computacional desenvolvido

No nível dos indicadores foi realizada a inserção dos valores de desempenho do POLO EAD. Com o modelo estruturado e alimentado o SAD permitiu visualizar a avaliação da GC, Figura 6. 


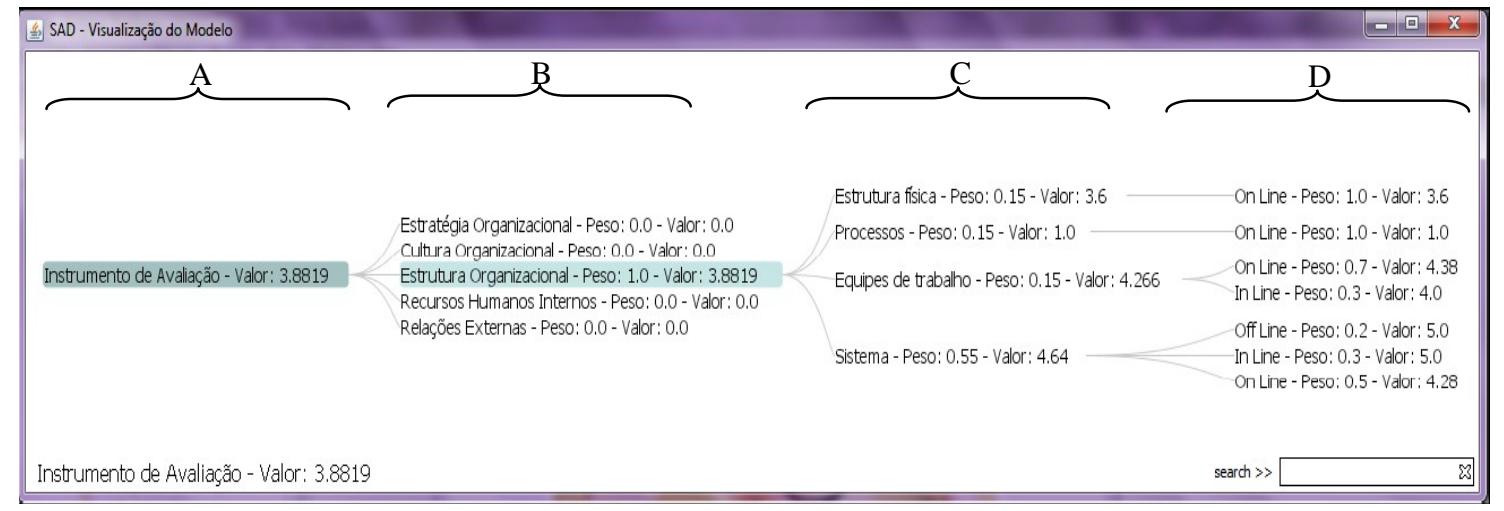

Figura 6 - Resultado no SAD da avaliação do POLO EAD.

Em relação à Figura 6, os elementos A, B, C e D representam os níveis de avaliação. No Nível A tem-se a pontuação final da avaliação, obtidos pela agregação das pontuações e pesos nos níveis hierarquicamente inferiores. No nível $\mathrm{B}$, têm-se as cinco percepções/pesos do modelo de avaliação da GC, acompanhadas de seus respectivos valores agregados a partir dos níveis inferiores. No nível $\mathrm{C}$, têm-se as preocupações/pesos do POLO EAD e os valores agregados a partir no nível de indicadores. No nível D, estão representados os indicadores/pesos e os valores de desempenho local.

\section{Considerações finais}

O mercado competitivo faz com que as organizações busquem cada vez mais informações que as auxiliem a direcionar esforços de aprendizagem e de gestão do conhecimento, a fim de inovar e melhorar seus desempenhos. Tais informações constituem elementos que auxiliam a (GC) nas organizações. No entanto, antes de se gerenciar o conhecimento é necessário que se avalie o desempenho, de modo a identificar pontos fortes e deficientes da organização, a fim de direcionar esforços.

Neste sentido, Deming (2003) destaca que em uma organização não é possível gerenciar o conhecimento sem primeiramente medi-lo, não se consegue medir $o$ conhecimento sem antes se ter um processo de avaliação. Por isso, os gestores necessitam de informações que os capacitem a tomar decisões baseados em fatos. A Avaliação de Desempenho (AD) pode então ser considerada uma atividade que auxilia a direcionar os esforços de aprendizagem e de gestão do conhecimento da organização, e que podem contribuir para melhorar seu desempenho.

Neste contexto, o presente estudo contribuiu ao propor o sistema computacional SAD que permite a estruturação de modelos de avaliação da gestão do conhecimento, de acordo com as especificidades organizacionais. O sistema desenvolvido utilizou tecnologias como Java que permite a portabilidade do sistema em vários tipos de plataforma, autenticação de usuário para tratar questões de sigilo dos dados, banco de dados MySQL para segurança e persistência dos dados, interface visual para facilitar a estruturação do modelo de avaliação da GC e o framework Prefuse que fornece uma apresentação gráfica do modelo, bem como dos pesos e valores da própria avaliação. Cabe ressaltar que, para validar o SAD, foi utilizado parcialmente o modelo de GC de uma organização de ensino à distância.

Em relação a estudos futuros, propõem-se: (a) a implementação de nova funcionalidade que permita realizar a análise histórica de avaliações de GC, de modo a comparar as avaliações de desempenho de uma organização, em tempos diferentes, 
permitindo identificar se as ações tomadas no período tiveram efeito positivo ou negativo no desempenho da organização; (b) alteração da interface do SAD de desktop para web, o que permitiria a publicação de resultados de avaliação pela Internet; e (c) modificação do projeto do SAD para permitir a alimentação automática de dados de desempenho.

\section{Referências}

BERZISA, S.; GRABIS, J. A framework for knowledge-based configuration of project management information systems. In: Proceedings of the 17th International Conference on Information and Software Technologies, Haiti. Kaunas University of Technology, Kaunas, 2011, p.31-38.

BOOCH, G.; RUMBAUGH, J.; JACOBSON, I. UML - Guia do Usuário. $2^{\text {a }}$ Ed. Editora Campus, 2006.

CARVALHO, R. B. Aplicações de Softwares de Gestão do Conhecimento: Tipologia e Usos. Dissertação (Mestrado) - Universidade Federal de Minas Gerais, Belo Horizonte, 2000.

CORMEN, T. H.; LEISERSON, C. E.; RIVEST, R. L.; STEIN, C. Algoritmos: teoria e prática. 2.a ed. Rio de Janeiro: Editora Campus/Elsevier, 2002.

DAVENPORT, T.; PRUSAK, L. Working Knowledge: how organizations manage what they know. Boston: Harvard Business School Press, 1998.

DEMING, W. E. Saia da crise. Tradução de Marcelo Alves Mendes. São Paulo: Editora Futura, 2003.

DRUCKER, P. F. As informações de que os executivos realmente precisam. In: Harvard business review. 3 ed. Rio de Janeiro: Campus, 2000.

IGARASHI, D.C.C.; IGARASHI, W.; CARDOSO, A.N.M.; SILVA, F.P.; NAKAYAMA, M.K. Operacionalização do modelo de Gestão do Conhecimento proposto por Terra (2005): uma análise segundo a ótica do gerenciamento. In: Revista ADMpg - gestão estratégica, 5,1, p. 33 - 43, 2012.

IGARASHI, D.C.C.; IGARASHI, W.; NAKAYAMA, M.K. Operacionalização do modelo de avaliação de aprendizagem organizacional proposto por Igarashi (2009) em um polo de ensino a distância. In: Renote, 8, 1, 2010.

IGARASHI, W.; IGARASHI, D.C.C.; VIEIRA, E.M.F; TODESCO, J.L. Operacionalização do modelo de avaliação de aprendizagem organizacional proposto por Igarashi (2009) em um polo de ensino a distância In: Cadernos Ebape, VI, 2, 6 - 18, 2008.

LARMAN, Craig, Utilizando UML e Padrões: uma introdução à análise e ao projeto orientado a objetos. 3. ed., Porto Alegre: Bookman, 2007.

LIAO, .S.H. Knowledge management technologies and applications-a literature review from 1995 to 2002 In: Expert Systems with Applications, 25(2), p. 155 - 64, 2003. 
LINS, M.E.; LOBO, M.E.C.; SILVA, A.C.M.; FISZMAN, R.W.J.P. O uso da Análise Envoltória de Dados (DEA) para avaliação de hospitais universitários brasileiros. In: Ciênc. saúde coletiva, Rio de Janeiro, v. 12, n. 4, Aug, 2007.

MELLO, J.C.C.B.S.; CLIMACO, J.C.N.; MEZA, L.A. Efficiency evaluation of a small number of DMUs: an approach based on Li and Reeves's model. In: Pesqui. Oper., Rio de Janeiro, v. 29, n. 1, Apr. 2009.

Rouse, W.B.; Dale, W. Compton, Systems engineering and management. In: Information-Knowledge-Systems Management, v.8 n.1-4, p.231-240, January, 2009.

Rouse, W.B.; Sage, A. P. Work, Workflow and Information Systems, IOS Press, Amsterdam, The Netherlands, 2007.

SALOMI, G.G.E.; MIGUEL, P.A.C.; ABACKERLI, A.J. SERVQUAL x SERVPERF: comparação entre instrumentos para avaliação da qualidade de serviços internos. Gest. Prod., São Carlos, v. 12, n. 2, Aug, 2005.

SANTOS, J.L.S.; LEOCADIO, L.; VARVAKIS, G. Gestão do Conhecimento como Processo: relação com tecnologias da informação e comunicação (TIC) e estratégia organizacional. São Paulo: SBGC. In: Congresso Brasileiro de GC, KM BRASIL,6. , 2007, São Paulo. Anais eletrônicos. CD-ROM, 2007.

SILBERSCHATZ, A.; KORTH, H.F.; SUDARSHAN, S. Sistema de Banco de Dados. $5^{\text {a }}$ edição. Tradução de Daniel Vieira. Rio de Janeiro: Elseviver, 2006.

SOMMERVILlE, I. Engenharia de Software. Tradução de Selma Shin Shimizu Melniko. 8 ed. São Paulo: Pearson Addion-Wesley, 2007.

SVEIBY, K. E. A Nova Riqueza das Organizações. Rio de Janeiro: Campus, 1998.

TAIT, T. F. C. Um Modelo de Arquitetura de Sistemas de Informação para o Setor Público: estudo em empresas estatais prestadoras de serviços de informática. 290 p. Tese (Doutorado) - Universidade Federal de Santa Catarina, Florianópolis, 2000.

TARAPANOFF, K. Inteligência organizacional e competitiva. Brasília: Editora Universidade de Brasília, 2001.

TERRA, J. C. C.; GORDON, C. Portais Corporativos: a revolução na gestão do conhecimento. São Paulo: Negócio Editora, 2002, 